\title{
Effectiveness of therapeutic suits and intensive suit trainings in individuals with cerebral palsy and other neurological disorders: a scoping review
}

\author{
DOI: https://doi.org/10.5114/pq.2020.100276
}

\author{
Ángel Luis Clemente Remón', Marta Agnieszka Panufnik ${ }^{2}$ \\ ${ }^{1}$ Faculty of Medicine and Health Sciences, University of Alcalá, Alcalá de Henares, Spain \\ ${ }^{2}$ Faculty of Education, University of Alcalá, Guadalajara, Spain
}

\begin{abstract}
Introduction. This review aims to find if there is any effectiveness in the application of therapeutic suits or intensive suit trainings or both together, among people with cerebral palsy and other pathologies.

Methods. Articles published from 1997 to 2019 were reviewed. Fifteen articles met the inclusion criteria that involved: English, Spanish, or Polish language; published studies; studies about the effectiveness of suit therapies regardless of the used protocol (Adeli suit, Therasuit, Penguin suit, or suit therapy); studies conducted among people with cerebral palsy or other neurological disorders regardless of age, type of cerebral palsy, and severity level.

Results. There were some positive effects in gross motor functions, functional gait, and joint mobility. However, the evidence was not clear and there were numerous factors that could have affected the results and produced methodological limitations. Conclusions. The findings justify further exploration of the potential of suit therapies in people with cerebral palsy and other neurological disorders.
\end{abstract}

Key words: cerebral palsy, Therasuit, Adeli suit, intensive training, intensive treatment, suit therapy

\section{Introduction}

Cerebral palsy (CP) is one of the most common causes of disability in children [1-7]. The incidence of CP is 2-2.5 per 1000 births $[5,8,9]$. CP is a term used to group a big heterogeneity of manifestations observed in people with brain disorders causing problems in motor abilities and other developmental aspects [4, 10-12]. In 2006, a committee of experts agreed on an international definition, as well as a classification for CP. According to Rosenbaum et al. [13], CP is a group of permanent developmental disorders related to movement and posture. These disorders cause an activity limitation attributed to nonprogressive disturbances that occurred in the developing foetal or infant brain. Motor disturbances usually are accompanied by perception disorders, cognitive and communicative difficulties, behavioural disturbances, and others $[8,14]$. In addition, epilepsy and secondary musculoskeletal problems are frequent.

According to the same authors and their guidelines in order to classify CP, 4 elements should be taken into consideration: type of motor disturbance, the presence or absence of other limitations or disorders, anatomical distribution and neuro-image findings, and the time of injury.

The rehabilitation for people with $\mathrm{CP}$ has focused its efforts on minimizing difficulties, promoting general function, and improving the quality of life $[7,15,16]$.

Treatments usually concentrate on several main goals. One of the most significant ones is preventing muscle weakness or, in other words, strengthening the muscular system. This is a very important aim because muscular weakness, altered muscle tone, deficient postural control [1, 17], limited passive joint mobility, and poor alignment [16] are common impairments in children and adults with CP [18]. Additionally, people with $\mathrm{CP}$, in comparison with healthy children and adults, move at a slower speed and show a poor balance [19].

The management of motor impairments resulting from CP consists of physical activity and a wide range of other therapies, more or less similar to physical therapy [11, 20]. There are numerous techniques applied in $\mathrm{CP}$, even though very few techniques or approaches have a real scientific base. Even, some of these treatments are considered as alternative or complementary therapies [10,13].

Suit therapy is one of these non-proved therapies. There are many names for this method: suit therapy, Penguin suit therapy, Adeli suit therapy, Polish suit therapy, Pediasuit therapy, Therasuit therapy, Spidersuit therapy [1]. Usually the designation alludes to protocols and suits [21].

The first suit was Adeli suit. It was developed by Russian scientists from the Soviet space program to help cosmonauts with their problems caused by the effects of zero gravity $[1,10$, $11,13,16,17,21-23]$. This orthotic device or dynamic orthosis consists of a vest, shorts, knee pieces, a head piece, and special shoes with hooks [1, 17, 18, 24, 25]. All these elements are linked, during the therapy, with elastic strings or bungee cords with varied levels of tension [14, 18, 24] to increase the body alignment and to provide proprioceptive information [3, 10, 11, 21, 23].

Mainly, the therapy with a suit, irrespective of the name, has 3 standards. The first one is the action of the suit per se; the second one has to do with the intensive training or therapy during several weeks; the last one is the requirement of active movement performed by the person with CP $[11,17,26]$.

Suit therapies are very commonly linked with intensive training. To define intensive training or intervention with $\mathrm{CP}$ is a difficult issue. Some researchers state that training applied more than 2 times per week can be considered inten-

Correspondence address: Ángel Luis Clemente Remón, Facultad de Medicina y Ciencias de la Salud de la Universidad de Alcalá, Ctra. Madrid-Barcelona. Km. 33,6, Alcalá de Henares, Madrid, Spain, e-mail: angel.clemente@uah.es 
sive [27]. However, the adjective 'intensive' refers to the duration of the training period (usually weeks), the frequency of trainings per week (days), and time spent each day (hours) [27]. The difficulty is related to the fact that a typical frequency of therapy or training for people with CP does not exist $[3,4]$. Some guidelines have been developed in order to establish rules concerning the frequency and intensity of treatments or trainings [28, 29]. Bailes et al. [28] state that an intensive training is performed 3-11 times per week and, for instance, Hanson et al. [29] indicate a frequency of 3-5 times per week.

When suit therapies or trainings are taken into consideration, the trainings usually consist of a 3-4-week program, implemented $4-5$ days per week, $2-4$ hours per day $[3,11$, $19,24,26,30]$.

Suit therapies have their specific characteristics, in addition to the intensity of the intervention. A traditional protocol of a suit therapy consists of stretching exercises, isolated strengthening activities for specific muscles, and functional training $[10,30]$. In spite of the protocol, trainings usually are not strictly determined, and there are adaptations depending on the person, the therapist, the goals, and many more.

Owing to this heterogeneity, it is difficult to determine the effectiveness of these trainings. Moreover, if the use of a suit is combined with the intensity of the training, both aspects are taken into consideration to measure the therapy success.

The aim of this review is to present the findings on the effectiveness of therapeutic suits and intensive suit trainings in people with CP and other neurological disorders on the basis of the research published so far. Comparisons of the characteristics of different types of interventions were conducted, as well as the characteristics that might explain the observed effect variances were investigated.

\section{Subjects and methods}

\section{Search strategy}

This scoping review focused on the literature pertaining to the effectiveness of exercises with a training suit among people with CP. An extensive literature search was conducted in the MEDLINE (PubMed), PEDro, Cochrane Library, and Web of Science databases, with no year, gender, age, or type of article restrictions; papers published until August 2019 were considered.

The key words used in the online search included 'Adeli suit,' 'Therasuit,' 'Penguin suit,' 'suit therapy,' and 'cerebral palsy'. The Boolean operator 'AND' was used to combine the search terms. The selection of these terms is justified because the therapeutic suits used in these kinds of therapies have different denominations depending on the registered trademark of the suit, even though all of them employ the same system. The term 'cerebral palsy' was selected to be sure that the therapies and suits were applied to treat this pathology or similar ones.

\section{Selection criteria}

The inclusion criteria were the following: (1) languages: English, Spanish, or Polish; (2) published studies; (3) studies on the effectiveness of suit therapies, regardless of the protocol: Adeli suit, Therasuit, Penguin suit, or suit therapy; (4) studies conducted in people with CP or other neurological disorders, regardless of age, type of $\mathrm{CP}$, and severity level. These criteria were set to collect as much information as it was possible about the suit therapies implemented in people with similar neurological disorders and to be able to compare these interventions.

The exclusion criteria involved: (1) articles without original research; (2) the application of another type of orthosis or orthotic equipment; (3) research without enough data or information concerning the protocols or measurements; (4) articles not available.

\section{Selection of studies and data extraction}

After reading titles and abstracts, studies that did not meet the inclusion criteria were removed. Also, duplicate studies were excluded.

Owing to the diversity of protocols and a limited number of studies, all protocols and experimental designs were con-

Table 1. Summary of the search and selection of the bibliography

\begin{tabular}{|c|c|c|c|c|}
\hline Database & Search strategy & $\begin{array}{l}\text { Total number } \\
\text { of results }\end{array}$ & $\begin{array}{l}\text { Results after elimination } \\
\text { of duplicate research }\end{array}$ & $\begin{array}{l}\text { Results after exclusion } \\
\text { criteria application }\end{array}$ \\
\hline \multirow{5}{*}{ PubMed } & Adeli suit & 13 & 13 & 8 \\
\hline & Therasuit & 6 & 6 & 4 \\
\hline & Suit therapy & 10 & 7 & 0 \\
\hline & Suit therapy and cerebral palsy & 32 & 17 & 12 \\
\hline & Penguin suit & 9 & 8 & 0 \\
\hline \multirow{2}{*}{ Web of Science } & Adeli suit & 13 & 4 & 1 \\
\hline & Therasuit & 2 & 0 & 0 \\
\hline \multirow{4}{*}{ PEDro } & Adeli suit & 3 & 0 & 0 \\
\hline & Therasuit & 1 & 0 & 0 \\
\hline & Suit therapy & 13 & 7 & 1 \\
\hline & Penguin suit & 0 & 0 & 0 \\
\hline \multirow{3}{*}{ Cochrane Library } & Adeli suit & 5 & 2 & 0 \\
\hline & Therasuit & 1 & 0 & 0 \\
\hline & Suit therapy & 3 & 1 & 0 \\
\hline
\end{tabular}



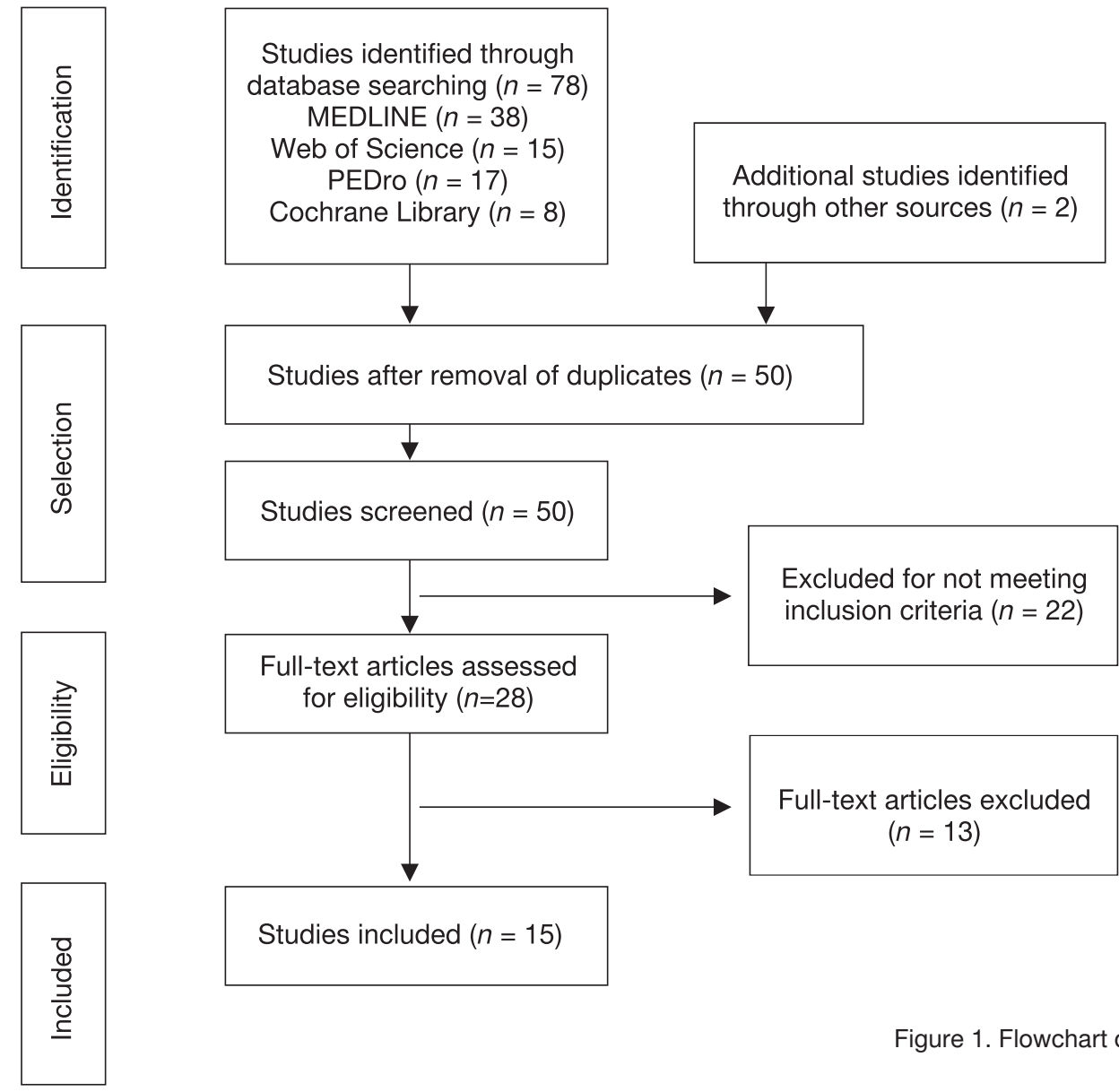

Studies included $(n=15)$ sidered. To identify other relevant articles, a manual search was conducted.

Two researchers read all the articles to determine which ones would be included in this review.

The searching and selection sequence are reflected in Table 1.

A specific strategy was followed to select as many appropriate studies as possible (Figure 1).

\section{Quality assessment}

Because of the diversity of the studies, 2 different tools were used to verify their quality. For randomized controlled trials, the Downs and Black [31] checklist was applied, as in other reviews [32-36]. For case reports, the Quality Assessment Tool for Case Series Studies was utilized from the National Heart, Lung, and Blood Institute [37], as in other research [38-41].

\section{Ethical approval}

The conducted research is not related to either human or animal use.

\section{Results}

From the initial 78 articles identified as potentially relevant studies, 15 met the criteria and were included in this review, as is shown in Figure 1. The included studies were published between 1997 and 2018.

There were a big variety of studies concerning the investigated issue: 1 retrospective study, 3 case reports, 5 single group designs, and 6 randomized clinical trials.
Figure 1. Flowchart of the article selection process

A big heterogeneity exists regarding the samples, protocols, and measurements. The inclusion and exclusion criteria are similar in most articles where these criteria could be found.

Data from the included studies were extracted by using a data extraction form involving: authors, study design, selection criteria, sample or study population, intervention protocol, measurements, and results. Table 2 shows a summary of these studies. Each research was numbered to facilitate the analysis in the successive sections and tables of this review.

\section{Randomized clinical trials}

The 6 randomized clinical trials presented a variability regarding all the surveyed aspects.

The inclusion and exclusion criteria were very similar. The samples consisted of children with different types of CP and diverse levels of severity. The subjects' age was between 4 and 12 years, although each study had a different range.

All samples performed an intensive exercise program in combination with a therapeutic suit. The training lasted from 3 weeks to 3 months, with the frequency of 3-7 days per week and the duration of the sessions from 30 minutes twice per day to 4 hours per day. Also, experimental and control group treatments were different. In 2 of the studies, the control groups received neurodevelopmental treatment (NDT). Also, in 2 of these studies, there was another control group: one received NDT + Adeli suit treatment (AST), and modified AST (MAST) was implemented in the other one. In 2 studies, the control group had a conventional therapy, and the study group followed a conventional therapy program with Thera- 







\begin{tabular}{|c|c|c|c|c|c|c|}
\hline 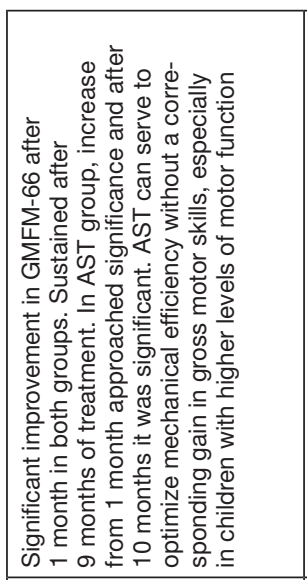 & 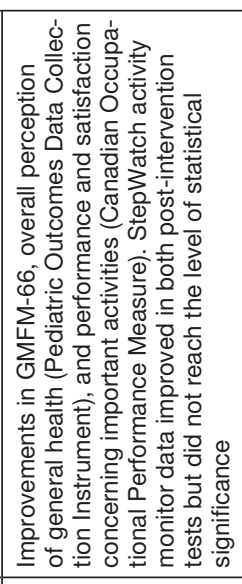 & 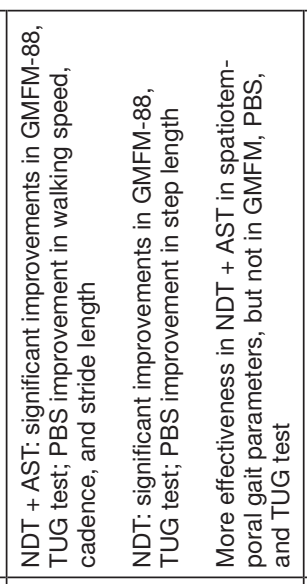 & 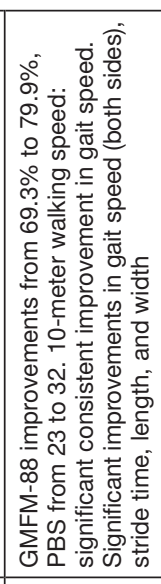 & 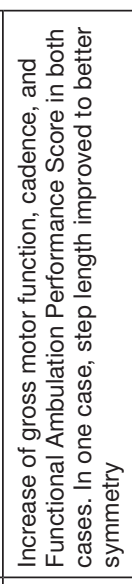 & 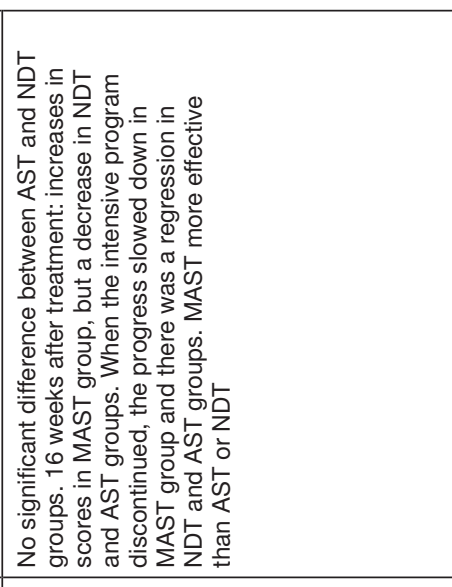 & \\
\hline 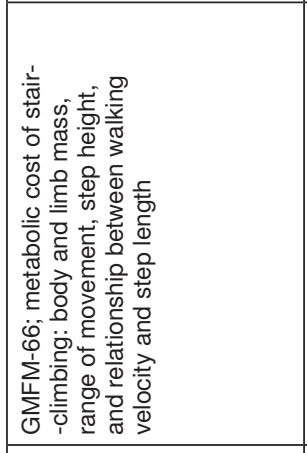 & 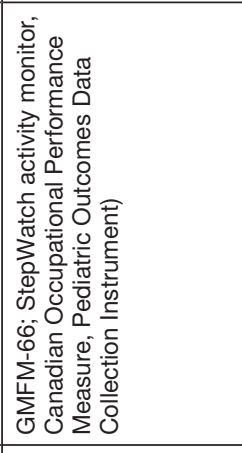 & 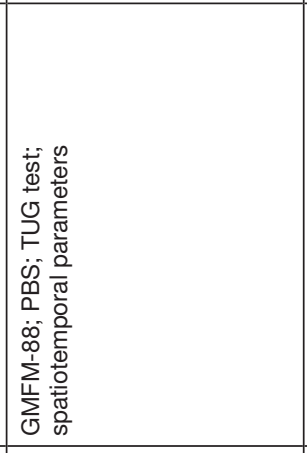 & 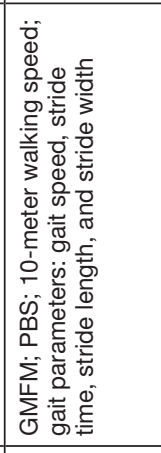 & 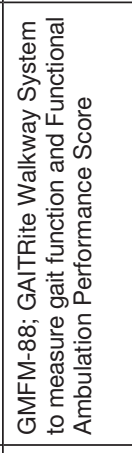 & $\sum_{i=1}^{\sum}$ & \\
\hline 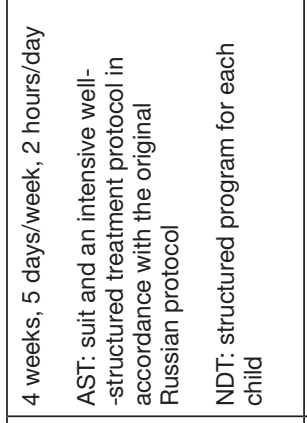 & 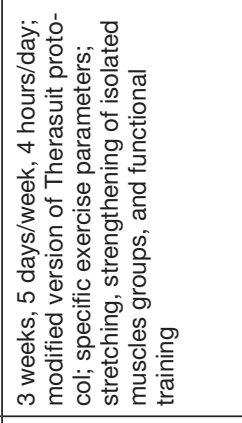 & 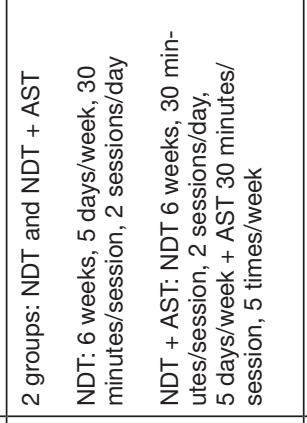 & 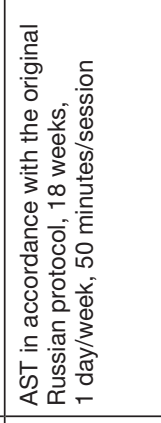 & 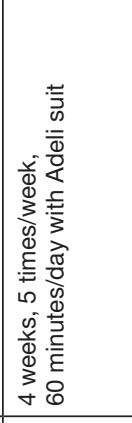 & 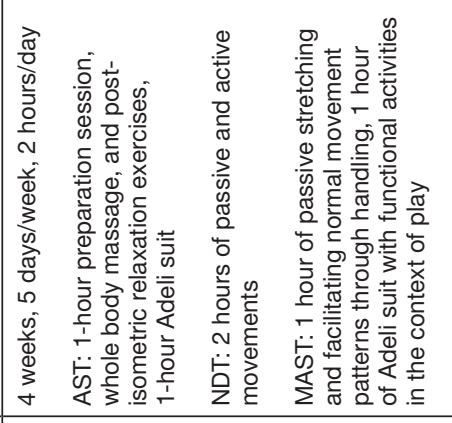 & 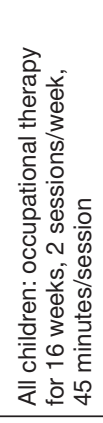 \\
\hline 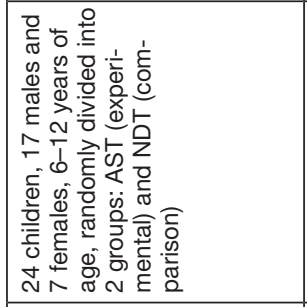 & 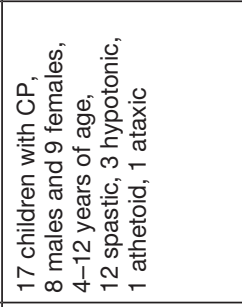 & 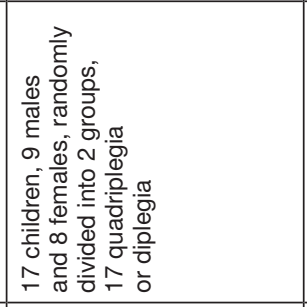 & 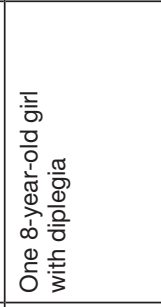 & 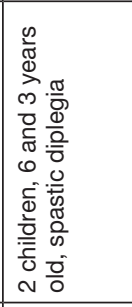 & 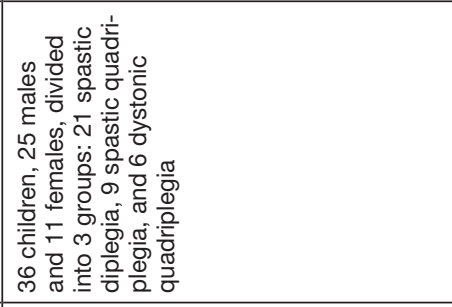 & \\
\hline 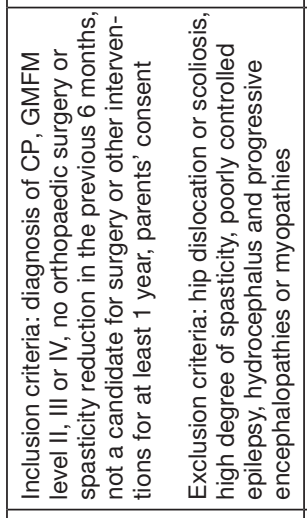 & 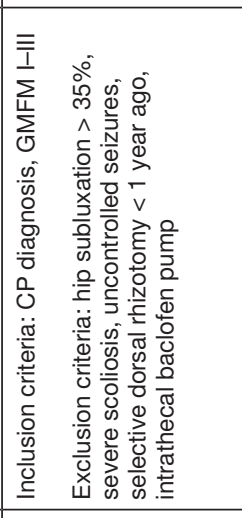 & 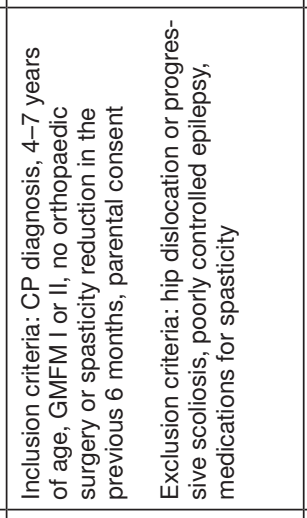 & 0 & 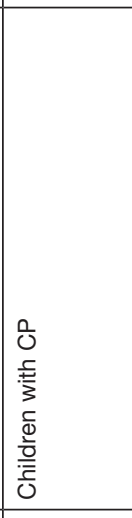 & 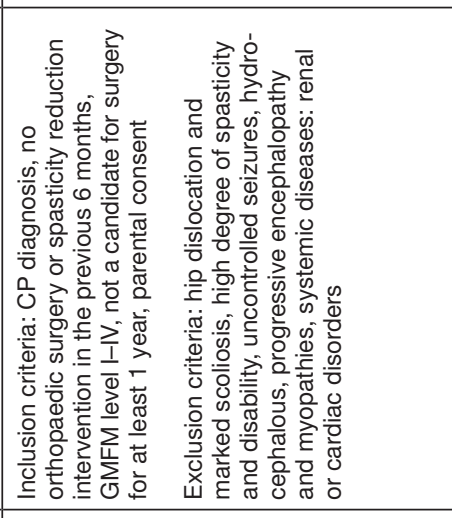 & \\
\hline 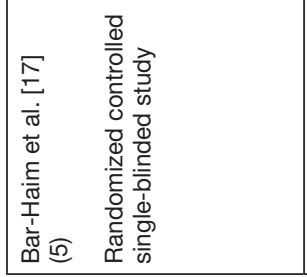 & 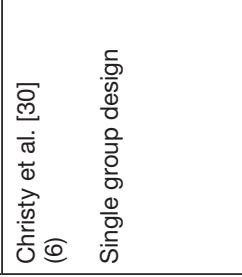 & 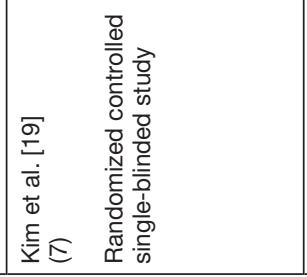 & 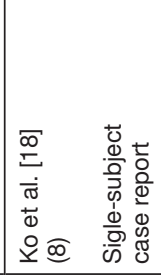 & 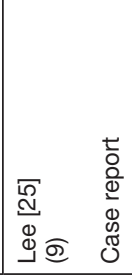 & 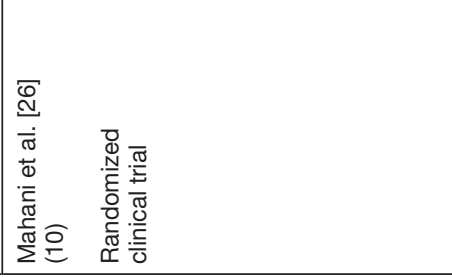 & \\
\hline
\end{tabular}




\begin{tabular}{|c|c|c|c|c|c|c|c|c|}
\hline \multicolumn{2}{|c|}{ 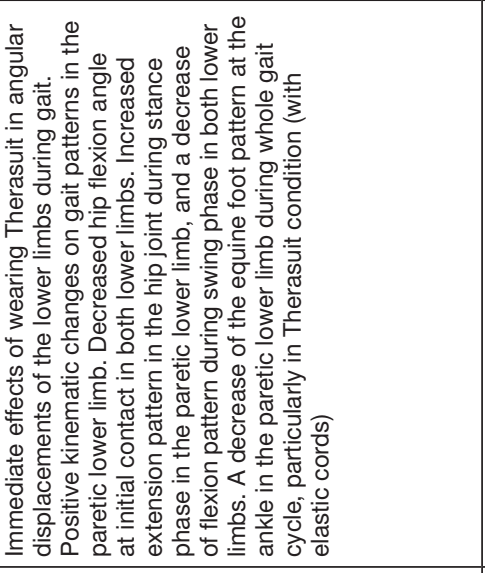 } & 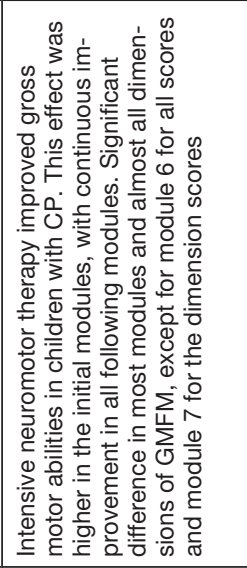 & \multicolumn{2}{|l|}{ 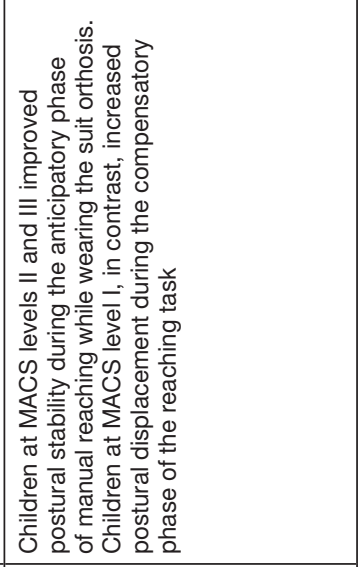 } & \multicolumn{2}{|c|}{ 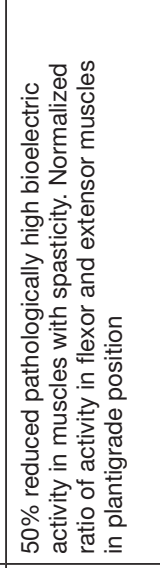 } & \multicolumn{2}{|c|}{ 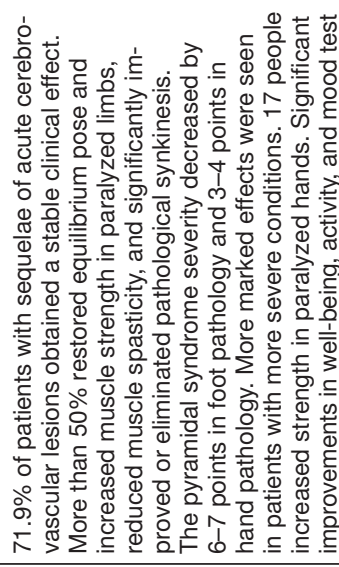 } \\
\hline \multicolumn{2}{|l|}{ 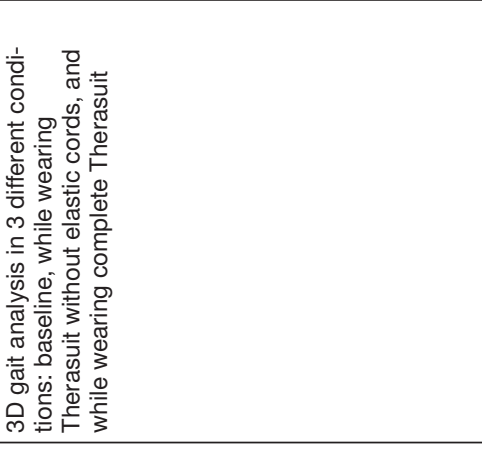 } & \begin{tabular}{|l|l}
$\infty$ \\
$\sum_{i=}^{\infty}$ \\
$\sum_{0}^{1}$ \\
\end{tabular} & \multicolumn{2}{|c|}{ 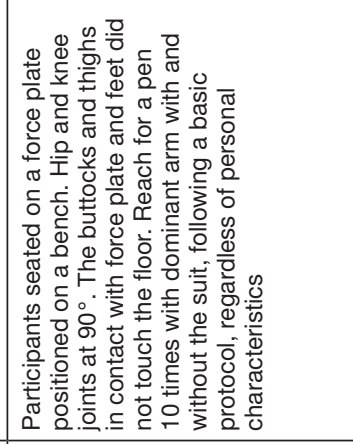 } & \multicolumn{2}{|c|}{ 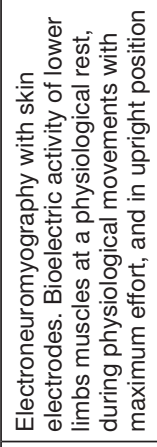 } & \multicolumn{2}{|l|}{ 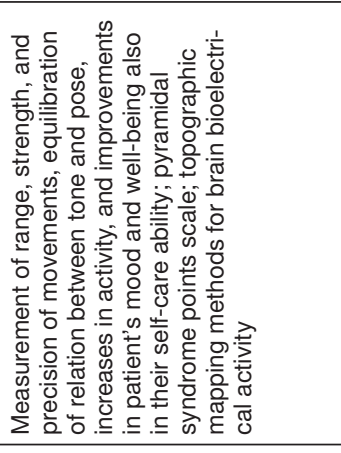 } \\
\hline 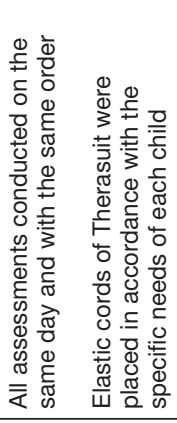 & 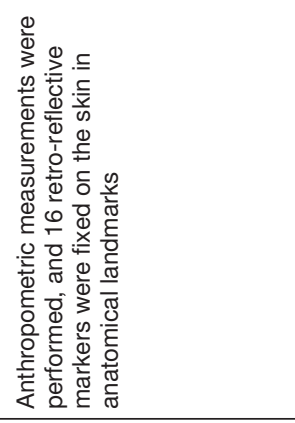 & 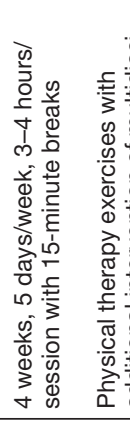 & 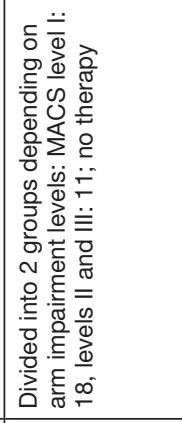 & & 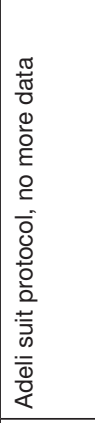 & & 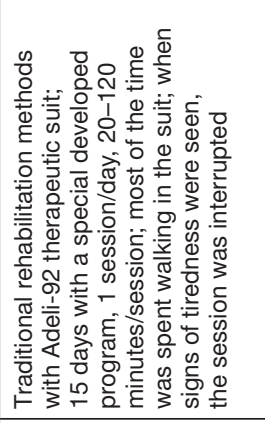 & \\
\hline 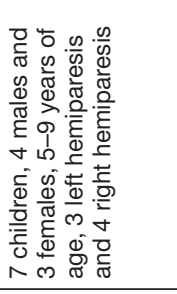 & & 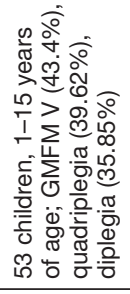 & 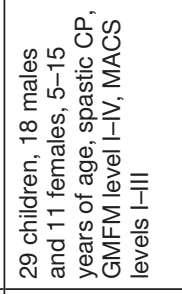 & & 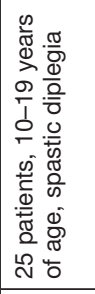 & & 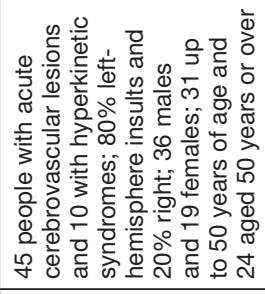 & \\
\hline 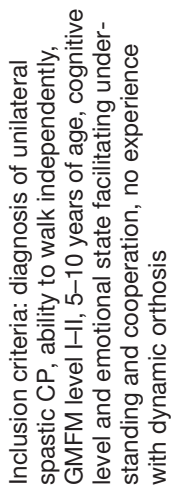 & 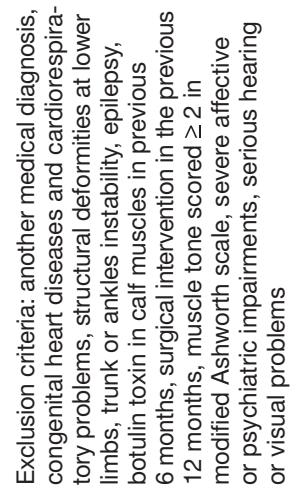 & 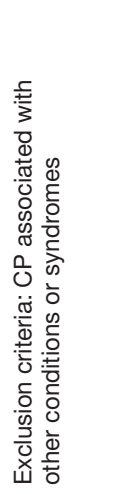 & 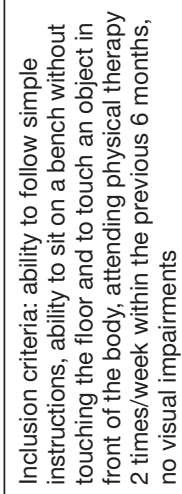 & 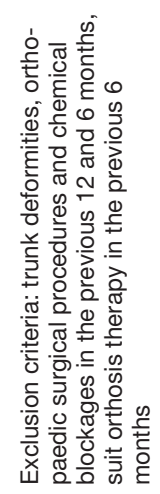 & 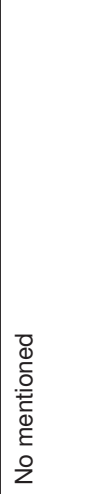 & & 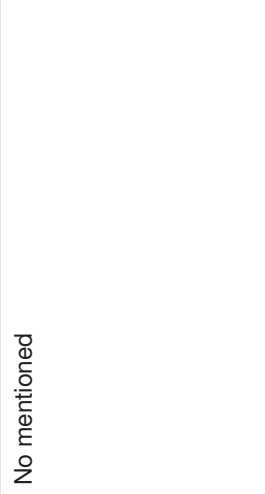 & \\
\hline 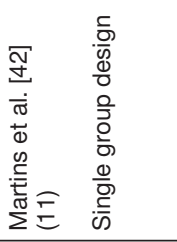 & & 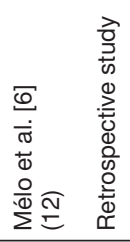 & 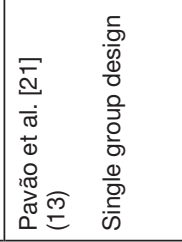 & & 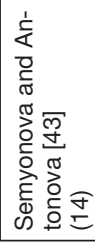 & & 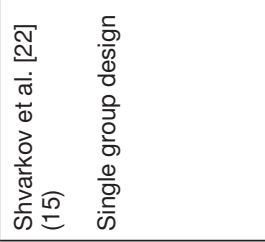 & \\
\hline
\end{tabular}


Table 3. Randomized clinical trials: methodological quality assessment checklist

\begin{tabular}{|c|c|c|c|c|c|c|}
\hline \multirow{2}{*}{ Checklist items } & \multicolumn{6}{|c|}{ Selected studies } \\
\hline & 1 & 2 & 4 & 5 & 7 & 10 \\
\hline \multicolumn{7}{|l|}{ Reporting } \\
\hline 1. Is the hypothesis/aim/objective of the study clearly described? & 1 & 1 & 1 & 1 & 1 & 1 \\
\hline $\begin{array}{l}\text { 2. Are the main outcomes to be measured clearly described in the introduction } \\
\text { or methods section? }\end{array}$ & 1 & 1 & 1 & 1 & 1 & 1 \\
\hline 3. Are the characteristics of the patients included in the study clearly described? & 1 & 1 & 1 & 1 & 1 & 1 \\
\hline 4. Are the interventions of interest clearly described? & 1 & 1 & 1 & 1 & 1 & 1 \\
\hline $\begin{array}{l}\text { 5. Are the distributions of principal confounders in each group of subjects } \\
\text { to be compared clearly described? }\end{array}$ & 0 & 0 & 2 & 0 & 0 & 0 \\
\hline 6. Are the main findings of the study clearly described? & 1 & 1 & 1 & 1 & 1 & 1 \\
\hline $\begin{array}{l}\text { 7. Does the study provide estimates of the random variability in the data } \\
\text { for main outcomes? }\end{array}$ & 1 & 1 & 1 & 1 & 1 & 1 \\
\hline $\begin{array}{l}\text { 8. Have all important adverse events that may be a consequence of the intervention } \\
\text { been reported? }\end{array}$ & 0 & 0 & 1 & 0 & 0 & 0 \\
\hline 9. Have the characteristic of patients lost to follow-up been described? & 0 & 1 & 1 & 1 & 1 & 1 \\
\hline $\begin{array}{l}\text { 10. Have actual probability values been reported for the main outcomes except } \\
\text { where the probability value is }<0.001 \text { ? }\end{array}$ & 1 & 1 & 1 & 1 & 1 & 1 \\
\hline $\begin{array}{l}\text { 11. Were the subjects asked to participate in the study representative of the entire } \\
\text { population from which they were recruited? }\end{array}$ & 0 & 0 & 0 & 0 & 0 & 0 \\
\hline $\begin{array}{l}\text { 12. Were those subjects who were prepared to participate representative of the entire } \\
\text { population from which they were recruited? }\end{array}$ & 0 & 0 & 0 & 0 & 0 & 0 \\
\hline $\begin{array}{l}\text { 13. Were the staff, places, and facilities where the patients were treated representative } \\
\text { of the treatment the majority of patients received? }\end{array}$ & 1 & 0 & 1 & 1 & 1 & 1 \\
\hline 14. Was an attempt made to blind study subjects to the intervention they received? & 0 & 0 & 1 & 0 & 0 & 0 \\
\hline $\begin{array}{l}\text { 15. Was an attempt made to blind those measuring the main outcomes } \\
\text { of the intervention? }\end{array}$ & 1 & 0 & 1 & 1 & 1 & 1 \\
\hline $\begin{array}{l}\text { 16. If any of the results of the study were based on 'data dredging,' was this } \\
\text { made clear? }\end{array}$ & 1 & 1 & 1 & 1 & 1 & 1 \\
\hline $\begin{array}{l}\text { 17. In trials or cohort studies, do the analyses adjust for different lengths of follow-up } \\
\text { of patients, or, in case-control studies, is the time period between the intervention } \\
\text { and outcome the same for cases and controls? }\end{array}$ & 0 & 1 & 1 & 1 & 1 & 1 \\
\hline 18. Were the statistical tests used to assess the main outcomes appropriate? & 1 & 1 & 1 & 1 & 1 & 1 \\
\hline 19. Was compliance with the interventions reliable? & 0 & 1 & 1 & 1 & 1 & 1 \\
\hline 20. Were the main outcome measures used accurate (valid and reliable)? & 1 & 1 & 1 & 1 & 1 & 1 \\
\hline $\begin{array}{l}\text { 21. Were the patients in different intervention groups (trials and cohort studies) or were } \\
\text { the cases and controls (case-control studies) recruited from the same population? }\end{array}$ & 1 & 1 & 1 & 1 & 1 & 1 \\
\hline $\begin{array}{l}\text { 22. Were study subjects in different intervention groups or were the cases and controls } \\
\text { recruited over the same period of time? }\end{array}$ & 1 & 1 & 1 & 1 & 1 & 1 \\
\hline 23. Were the study subjects randomized to intervention groups? & 1 & 1 & 1 & 1 & 1 & 1 \\
\hline $\begin{array}{l}\text { 24. Was the randomized intervention assignment concealed from both patients } \\
\text { and health care staff until recruitment was complete and irrevocable? }\end{array}$ & 0 & 0 & 0 & 0 & 0 & 0 \\
\hline $\begin{array}{l}\text { 25. Was there adequate adjustment for confounding in the analyses from which } \\
\text { the main findings were drawn? }\end{array}$ & 0 & 0 & 1 & 0 & 0 & 0 \\
\hline 26. Were losses of patients to follow-up taken into account? & 0 & 1 & 1 & 1 & 1 & 1 \\
\hline \multicolumn{7}{|l|}{ Power } \\
\hline $\begin{array}{l}\text { 27. Did the study have sufficient power to detect a clinically important effect where the } \\
\text { probability value for a difference being due to chance is }<5 \% \text { ? }\end{array}$ & 1 & 1 & 1 & 1 & 1 & 1 \\
\hline Total score & 16 & 18 & 25 & 20 & 20 & 20 \\
\hline
\end{tabular}

Yes $=1$ point, no or unable to determine $=0$ points. For question 5 : yes $=2$ points, partially $=1$ point, no $=0$ points.

Results ranges: excellent (26-28), good (20-25), fair (15-19), and poor ( $\leq 14)$ 
Table 4. Quality Assessment Tool for Case Series Studies (National Heart, Lung, and Blood Institute [37])

\begin{tabular}{|c|c|c|c|c|c|c|c|c|c|}
\hline \multirow{2}{*}{ Criteria } & \multicolumn{9}{|c|}{ Selected studies } \\
\hline & 3 & 6 & 8 & 9 & 11 & 12 & 13 & 14 & 15 \\
\hline 1. Was the study question or objective clearly stated? & Yes & Yes & Yes & Yes & Yes & Yes & Yes & Yes & Yes \\
\hline $\begin{array}{l}\text { 2. Was the study population clearly and fully described, } \\
\text { including a case definition? }\end{array}$ & Yes & Yes & Yes & Yes & Yes & Yes & Yes & No & Yes \\
\hline 3. Were the cases consecutive? & NA & NA & NA & NA & NA & No & NA & NA & NA \\
\hline 4. Were the subjects comparable? & Yes & Yes & NA & NA & Yes & Yes & Yes & CD & Yes \\
\hline 5. Was the intervention clearly described? & Yes & No & Yes & Yes & Yes & Yes & Yes & No & No \\
\hline $\begin{array}{l}\text { 6. Were the outcome measures clearly defined, valid, reliable, } \\
\text { and implemented consistently across all study participants? }\end{array}$ & Yes & Yes & Yes & Yes & Yes & Yes & Yes & Yes & No \\
\hline 7. Was the length of follow-up adequate? & $C D$ & CD & Yes & $\mathrm{CD}$ & Yes & $C D$ & Yes & CD & $C D$ \\
\hline 8. Were the statistical methods well described? & Yes & Yes & Yes & NA & Yes & Yes & Yes & No & No \\
\hline 9. Were the results well described? & Yes & Yes & Yes & Yes & Yes & Yes & Yes & Yes & Yes \\
\hline
\end{tabular}

Possible answers: Yes, No, CD - cannot determine, NR - not reported, NA - not applicable. For each item where 'No' was selected, a potential risk of bias can be considered. 'Cannot determine' and 'not reported' were also noted as representing potential flaws.

suit or a modified suit therapy. In the last trial, a comparison was made with the same protocol but using the suit with and without bungee cords.

Regarding the measurements, in 5 of the studies, Gross Motor Function Measure (GMFM) was applied exclusively or in combination with other scales like Pediatric Evaluation of Disability Inventory (PEDI), Pediatric Balance Scale (PBS), or the Timed Up and Go (TUG) test. Also, some studies took into consideration other parameters, like spatiotemporal or metabolic cost, and 1 study measured back geometry.

In all research, improvements were seen after the application of the treatment protocols. Alagesan and Shetty [1] established higher efficacy of conventional therapy in combination with suit therapy. Azab and Hamed [2] observed an improvement in back geometry in children from both groups. Bailes et al. [3] did not demonstrate any improvement in motor function in children wearing Therasuit in comparison with those wearing a control suit. Bar-Haim et al. [17] suggested that AST optimized mechanical efficiency in children with higher levels of motor function, without a corresponding gain in gross motor skills. Kim et al. [19] found a higher improvement in spatiotemporal gait parameters in the AST/ NDT group, but no differences between groups in GMFM, PBS, or TUG test. Finally, Mahani et al. [26] noticed a greater effectiveness of MAST in comparison with AST and NDT.

The selected randomized clinical trials were surveyed in order to determine their quality by using a checklist for the assessment of the methodological quality of both randomized and non-randomized studies of health care interventions proposed by Downs and Black [31] in its modified version. The results are shown in Table 3 . Using this checklist it can be determined that Bailes et al. [3] is good and the rest of them are fair, taking into consideration the result ranges.

Case reports, single group designs, and the retrospective study

Nine studies were included in this section.

The samples were 1, 2 (2 studies), 7, 17, 25, 29, 45, and 53 subjects. In all studies, the sample involved children with different types of $\mathrm{CP}$, except in the last one, in which the study population consisted of adults after acute cerebrovascular lesions or with hyperkinetic syndromes.
Seven studies with treatment protocols were considered and 2 without treatment, only measurements. The treatment protocols were varied; nevertheless, 4 of them described a 3-4-week intervention with trainings on 5 days per week. Within these protocols, 3 had 3-4 hours of training per day, and there was only 1 hour in 1 study. Another protocol involved 18 consecutive weeks with one 50 -minute long session once per week. There was 1 study with a 15-day treatment, 20 120 minutes per day, comprising traditional rehabilitation with an Adeli suit. The last treatment was very poorly described, the Adeli suit protocol was performed without any additional data.

The measurement tools were GMFM in 5 studies exclusively or in combination with other tests, like PEDI and 3D gait analysis [3]; StepWatch activity monitor, Canadian Occupational Performance Measure, and Pediatric Outcomes Data Collection Instrument [30]; and PBS, 10-meter walking speed and gait parameters [18]. Semyonova and Antonova [43] used electroneuromyography and Shvarkov et al. [22] measured the range, strength, and precision of movements, equilibration, and patients' mood.

Although all of the studies showed improvements in measurements, almost all authors stated that further studies were necessary to confirm their findings.

There were studies without treatment protocols. These compared measurements in conditions with and without suits. These measurements applied a 3D gait analysis to evaluate the effects on gait, a force plate to assess postural stability. Martins et al. [42] concluded that some positive immediate effects were seen in gait kinematics when a suit orthosis was used, and Pavão et al. [21] observed that the suit improved postural stability in children with Manual Ability Classification System levels II and III during anticipatory postural adjustments.

To evaluate the quality of these studies, a quality assessment tool from the National Heart, Lung, and Blood Institute was used. In this tool, for each item where 'no' was selected, a potential risk of bias can be considered that could be introduced by that flaw in the study design or implementation. 'Cannot determine' and 'not reported' were also noted as representing potential flaws. The results are reflected in Table 4. There are similarities among most of the articles. We can consider the majority of them as a 'good' research; however, a lower quality can be observed in studies 14 and 15 [22, 43]. 


\section{Discussion}

This scoping review examined the current evidence of effects of therapeutic suits (Therasuit or Adeli suit), associated or not with intensive training protocols.

There is not much investigation accessible on this topic. Nevertheless, in most studies selected for this review, improvements in the measurements were observed. However, the evidence is not clear and there are numerous factors that can affect the results and produce methodological limitations.

The first limitation could be the fact that studies with statistically significant or positive findings are more likely to be published than trials with nonsignificant findings; then, the validity of a review conclusions can be threatened [44, 45].

Also, a limitation is the shortage of evidence and large heterogeneity in the existing trials regarding the type of study and their improvable quality, especially in oldest studies.

The samples were another limitation. They were very small (the largest one involved 55 people [22]) and there was a big a variability among them. The samples were comprised of children of different ages, as well as adults in one of the studies, with very diverse characteristics: type of CP, severity, and GMFM level. This is an important point because age could influence the level of gross motor function and the improvement potential.

Inclusion and exclusion criteria, when mentioned, were very constrained; this aspect is also a limitation because $\mathrm{CP}$ and other similar neurological disorders are very commonly associated with other problems, for instance, cognitive, sensorial, or behavioural disorders [8, 13, 14]. Studies should preferably focus on more real samples to test the effectiveness of the treatment in most common patients and enable result generalization.

Regarding the measurements, the most frequently used one was GMFM in combination with other tests or examinations in some research. The majority of these tests and measurements are useful for quantitative evaluations, even though, especially in the most affected people, these improvements could be difficult to measure.

The diversity in the protocols, treatments, and comparisons among groups in each study was another limitation. It is complex to determine, in some of the studies, if the results were due to the suit, the protocol, or combination of both.

Case reports determined improvements in gross motor function, functional gait, and joint movements. However, all the researchers stated that more studies were needed to confirm their results and to clarify if the improvement was due to the suit by itself, the intensive training, or combination of both. Some studies seemed to show that the suit by itself produced improvement in postural stability and gait kinematics in children wearing the suits without any training protocol [21, 42]. Also the suit combined with intensive training protocols apparently produced better results than the intensive training in isolation [1], even though in other similar studies [2] the results were parallel in both groups, with and without suits.

Another important aspect to determine is if the effectiveness of the suit in combination with treatment protocols is always similar or if there are different factors that can affect it. Mélo et al. [6] mentioned that there were improvements in GMFM in all children in almost every module of treatment, even though they observed that the improvements were greater in the first modules than in the last ones of each child. This could be due to the age or the patients' getting used to the suit, the treatment, or both.
Therapists' opinions were not compiled in any of the studies and this could be an important dimension to take into account. The suit improved the alignment of patients by itself $[3,10,16,21,23]$ and it would be a good assistance for the therapists to reduce their effort and fatigue during working time. If it were demonstrated, probably the use of the suit during intensive treatments could have a reasonable explanation, regardless the suit effects on patients.

The criteria for selecting the studies were met, with a language restriction. Only studies in English, Spanish, or Polish were included, which resulted in the exclusion of some, possibly pertinent, studies in Russian.

Finally, a good quality assessment tool for all studies was not found because of the diversity in their structure and format. Therefore, 2 different tools were necessary.

Owing to these limitations, the conclusions of the review were seriously limited, even though there are several interesting issues for future investigations.

Further research is necessary to confirm the effectiveness of this type of treatment, reducing the risk of bias and other limitations that have been highlighted before.

Also, a very important aspect that needs to be correctly measured is the quality of life in people with $\mathrm{CP}$ and the effects of the therapeutic intervention on this aspect. The measurement of the patients' quality of life reflects a holistic approach to patients with CP [46] and can be one of the main indicators of the effectiveness of the therapeutic methods [7]. Quality of life evaluation could provide important information about the patient's physical and mental state, necessary to perceive problems and to take actions aimed at solving them [47].

In connection with the previous idea, measuring the effectiveness of therapeutic suits and intensive trainings can be related to the improvement in other aspects that are usually affected in CP patients, like chronic constipation. Some of the several reasons for constipation are poor diet and decreased intestinal motility [48]; very likely, an intensive treatment with a therapeutic suit could help solve both these problems.

\section{Conclusions}

The results show that, nowadays, the evidence of the effectiveness of therapeutic suits like Therasuit or Adeli suit is weak. Therefore, more experimental studies with wider samples are required to confirm the results or to clarify the reasons for the method effectiveness.

Samples should be more homogeneous in each investigation, even though exclusion criteria should be less restrictive, including children with several disorders.

Additionally, it would be relevant to determine the impact of suits and intensive treatments in isolation.

Therapists' opinions should be surveyed to reinforce the usefulness of the suit, not only regarding the improvements in children's development, but also in the facilitation of the therapists' work.

Finally, the development of adequate assessment tools to test the quality of different types of trials could be interesting.

\section{Disclosure statement}

No author has any financial interest or received any financial benefit from this research.

\section{Conflict of interest}

The authors state no conflict of interest. 


\section{References}

1. Alagesan J, Shetty A. Effect od modified suit therapy in spastic diplegic cerebral palsy - a single blinded randomized controlled trial. Online J Health Allied Sci. 2010; 9(4):14.

2. Azab ASR, Hamed SA. Effect of suit therapy on back geometry in spastic diplegic cerebral palsied children. J Am Sci. 2014;10(10):245-251; doi: 10.13140/RG.2.1. 3806.2161.

3. Bailes AF, Greve K, Burch CK, Reder R, Lin L, Huth MM. The effect of suit wear during an intensive therapy program in children with cerebral palsy. Pediatr Phys Ther. 2011;23(2):136-142; doi: 10.1097/PEP.0b013e318218e f58.

4. Elgawish MH, Zakaria MA. The effectiveness of intensive versus standard physical therapy for motor progress in children with spastic cerebral palsy. Egypt Rheumatol Rehabil. 2015;42(1):1-6; doi: 10.4103/1110-161X.155622.

5. Eunson P. Aetiology and epidemiology of cerebral palsy. Paediatr Child Health. 2012;22(9):361-366; doi: 10.1016/ j.paed.2012.05.008.

6. Mélo TR, Yamaguchi B, Chiarello CR, Szczypior Costin AC, Erthal V, Israel VL, et al. Intensive neuromotor therapy with suit improves motor gross function in cerebral palsy: a Brazilian study. Motricidade. 2017;13(4):5461; doi: 10.6063/motricidade.13699.

7. Tatla SK, Sauve K, Virji-Babul N, Holsti L, Butler C, van der Loos HFM. Evidence for outcomes of motivational rehabilitation interventions for children and adolescents with cerebral palsy: an American Academy for Cerebral Palsy and Developmental Medicine systematic review. Dev Med Child Neurol. 2013;55(7):593-601; doi: 10.1111/ dmcn.12147.

8. Colver A, Fairhurst C, Pharaoh POD. Cerebral palsy. Lancet. 2014;383(9924):1240-1249; doi: 10.1016/S0140 -6736(13)61835-8.

9. MacLennan AH, Thompson SC, Gecz J. Cerebral palsy: causes, pathways, and the role of genetic variants. Am J Obstet Gynecol. 2015;213(6):779-788; doi: 10.1016/j. ajog.2015.05.034.

10. Liptak GS. Complementary and alternative therapies for cerebral palsy. Ment Retard Dev Disabil Res Rev. 2005; 11(2):156-163; doi: 10.1002/mrdd.20066.

11. Martins E, Cordovil R, Oliveira R, Letras S, Lourenço S, Pereira I, et al. Efficacy of suit therapy on functioning in children and adolescents with cerebral palsy: a systematic review and meta-analysis. Dev Med Child Neurol. 2016;58(4):348-360; doi: 10.1111/dmcn.12988.

12. Rosenbaum P. Controversial treatment of spasticity: exploring alternative therapies for motor function in children with cerebral palsy. J Child Neurol. 2003;18(Suppl. 1):S89-S94; doi: 10.1177/0883073803018001S0101.

13. Rosenbaum P, Paneth N, Leviton A, Goldstein M, Bax M, Damiano D, et al. A report: the definition and classification of cerebral palsy April 2006. Dev Med Child Neurol Suppl. 2007;109:8-14; doi: 10.1111/j.1469-8749.2007. tb12610.x.

14. Smith M, Kurian MA. The medical management of cerebral palsy. Paediatr Child Health. 2012;22(9):372-376; doi: 10.1016/j.paed.2012.04.002.

15. Almeida KM, Fonseca ST, Figueiredo PRP, Aquino AA, Mancini MC. Effects of interventions with therapeutic suits (clothing) on impairments and functional limitations of children with cerebral palsy: a systematic review. Braz J Phys Ther. 2017;21(5):307-320; doi: 10.1016/j.bjpt. 2017.06.009.
16. Bailes AF, Greve K, Schmitt LC. Changes in two children with cerebral palsy after intensive suit therapy: a case report. Pediatr Phys Ther. 2010;22(1):76-85; doi: 10.1097/ PEP.0b013e3181cbf224.

17. Bar-Haim S, Harries N, Belokopytov M, Frank A, Copeliovitch L, Kaplanski J, et al. Comparison of efficacy of Adeli suit and neurodevelopmental treatments in children with cerebral palsy. Dev Med Child Neurol. 2006;48(5): 325-330; doi: 10.1017/S0012162206000727.

18. Ko M-S, Lee J-A, Kang S-Y, Jeon H-S. Effect of Adeli suit treatment on gait in a child with cerebral palsy: a singlesubject report. Physiother Theory Pract. 2014;31(4):275282; doi: 10.3109/09593985.2014.996307.

19. Kim M-R, Lee B-H, Park D-S. Effects of combined Adeli suit and neurodevelopmental treatment in children with spastic cerebral palsy with gross motor function classification system levels I and II. Hong Kong Physiother J. 2016;34:10-18; doi: 10.1016/j.hkpj.2015.09.036.

20. Hombergen SP, Huisstede BM, Streur MF, Stam HJ, Slaman J, Bussmann JB, et al. Impact of cerebral palsy on health-related physical fitness in adults: systematic review. Arch Phys Med Rehabil. 2012;93(5):871-881; doi: 10.1016/j.apmr.2011.11.032.

21. Pavão S, Visicato LP, da Costa CSN, de Campos AC, Rocha NACF. Effects of suit-orthosis on postural adjustments during seated reaching task in children with cerebral palsy. Pediatr Phys Ther. 2018;30(3):231-237; doi: 10.1097/PEP.0000000000000519.

22. Shvarkov SB, Davydov OS, Kuuz RA, Aipova TR, Vein AM. New approaches to the rehabilitation of patients with neurological movement defects. Neurosci Behav Physiol. 1997;27(6):644-647; doi: 10.1007/bf02461921.

23. Solopova IA, Moshonkina TR, Umnov VV, Vissarionov SV, Baindurashvili AG, Gerasimenko YP. Neurorehabilitation of patients with cerebral palsy. Hum Physiol. 2015;41(4): 448-454; doi: 10.1134/S0362119715040155.

24. Turner AE. The efficacy of Adeli suit treatment in children with cerebral palsy. Dev Med Child Neurol. 2006;48(5): 324; doi: 10.1017/S0012162206000715.

25. Lee B-H. Clinical usefulness of Adeli suit therapy for improving gait function in children with spastic cerebral palsy: a case study. J Phys Ther Sci. 2016;28(6):19491952; doi: 10.1589/jpts.28.1949.

26. Mahani MK, Karimloo M, Amirsalari S. Effects of modified Adeli suit therapy on improvement of gross motor function in children with cerebral palsy. Hong Kong $J$ Occup Ther. 2011;21(1):9-14; doi: 10.1016/j.hkjot.2011. 05.001.

27. Myrhaug HT, Østensjø S, Larun L, Odgaard-Jensen J, Jahnsen R. Intensive training of motor function and functional skills among young children with cerebral palsy: a systematic review and meta-analysis. BMC Pediatr. 2014;14:292; doi: 10.1186/s12887-014-0292-5.

28. Bailes AF, Reder R, Burc C. Development of guidelines for determining frequency of therapy services in a pediatric medical setting. Pediatr Phys Ther. 2008;20(2):194198; doi: 10.1097/PEP.0b013e3181728a7b.

29. Hanson H, Harrington AT, Nixon-Cave K. Implementing treatment frequency and duration guidelines in a hospital-based pediatric outpatient setting: administrative case report. Phys Ther. 2015;95(4):678-684; doi: 10.2522/ ptj.20130360.

30. Christy JB, Chapman CG, Murphy P. The effect of intense physical therapy for children with cerebral palsy. J Pediatr Rehabil Med. 2012;5(3):159-170; doi: 10.3233/ PRM-2012-0208. 
31. Downs SH, Black N. The feasibility of creating a checklist for the assessment of the methodological quality both of randomised and non-randomised studies of health care interventions. J Epidemiol Community Health. 1998; 52(6):377-384; doi: 10.1136/jech.52.6.377.

32. Cascia N, Picha K, Hettrich CM, Uhl TL. Considerations of conservative treatment after a partial ulnar collateral ligament injury in overhead athletes: a systematic review. Sports Health. 2019;11(4):367-374; doi: 10.1177/19417 38119853589.

33. Chakravorty A, Mobbs RJ, Anderson DB, Rooke K, Phan K, Yoong N, et al. The role of wearable devices and objective gait analysis for the assessment and monitoring of patients with lumbar spinal stenosis: systematic review. BMC Musculoskelet Disord. 2019;20(1):288; doi: 10.1186/s12891-019-2663-4.

34. Mallari B, Spaeth EK, Goh H, Boyd BS. Virtual reality as an analgesic for acute and chronic pain in adults: a systematic review and meta-analysis. J Pain Res. 2019;12: 2053-2085; doi: 10.2147/JPR.S200498.

35. Szeszulski J, Lorenzo E, Shaibi GQ, Buman MP, VegaLópez S, Hooker SP, et al. Effectiveness of early care and education center-based interventions for improving cardiovascular fitness in early childhood: a systematic review and meta-analysis. Prev Med Rep. 2019;15:100915; doi: 10.1016/j.pmedr.2019.100915.

36. Szkwara JM, Milne N, Hing W, Pope R. Effectiveness, feasibility, and acceptability of dynamic elastomeric fabric orthoses (DEFO) for managing pain, functional capacity, and quality of life during prenatal and postnatal care: a systematic review. Int J Environ Res Public Health. 2019;16(13):2408; doi: 10.3390/ijerph16132408.

37. National Heart, Lung, and Blood Institute. Study quality assessment tools. Available from: https://www.nhlbi.nih. gov/health-topics/study-quality-assessment-tools.

38. Conolly MP, Haitsma G, Hernández AV, Vidal JE. Systematic review and meta-analysis of secondary prophylaxis for prevention of HIV-related toxoplasmic encephalitis relapse using trimethoprim-sulfamethoxazole. Pathog Glob Health. 2017;111(6):327-331; doi: 10.1080/20477 724.2017.1377974.

39. Diez-Fernández A, Álvarez-Bueno C, Martínez-Vizcaíno V, Sotos-Prieto M, Recio-Rodríguez JI, Cavero-Redondo I. Total dairy, cheese and milk intake and arterial stiffness: a systematic review and meta-analysis of crosssectional studies. Nutrients. 2019;11(4):741; doi: 10.3390/ nu11040741.

40. Harris JA, Moniz MH, lott B, Power R, Griggs JJ. Obesity and the receipt of influenza and pneumococcal vaccination: a systematic review and meta-analysis. BMC Obes. 2016;3:24; doi: 10.1186/s40608-016-0105-5.

41. Koppen IJN, Kuizenga-Wessel S, Saps M, Di Lorenzo C, Benninga MA, van Etten-Jamaludin FS, et al. Functional defecation disorders and excessive body weight: a systematic review. Pediatrics. 2016;138(3):e20161417; doi: 10.1542/peds.2016-1417.

42. Martins E, Cordovil R, Oliveira R, Pinho J, Diniz A, Vaz JR. The immediate effects of a dynamic orthosis on gait patterns in children with unilateral spastic cerebral palsy: a kinematic analysis. Front Pediatr. 2019;7:42; doi: 10.3389/fped.2019.00042.

43. Semyonova KA, Antonova LV. The influence of the LK-92 "Adeli" treatment loading suit on electro-neuro-myographic characteristics in patients with infantile cerebral paralysis. Zh Nevrol Psikhiatr Im S S Korsakova.
44. Hopewell S, Loudon K, Clarke MJ, Oxman AD, Dickersin K. Publication bias in clinical trials due to statistical significance or direction of trial results. Cochrane Database SystRev.2009;1(1):MR000006;doi:10.1002/14651858. MR000006.pub3.

45. Dwan K, Altman DG, Arnaiz JA, Bloom J, Chan A-W, Cronin E, et al. Systematic review of the empirical evidence of study publication bias and outcome reporting bias. PLoS One. 2008;28:3(8):e3081; doi: 10.1371/ journal.pone.0003081.

46. Lewicka M, Kurylak A. Assessment of quality of life in patients with cerebral palsy. Pediatr Pol. 2019;94(2): 119-127; doi: 10.5114/polp.2019.85041.

47. Lewicka M, Kurylak A. The quality of life of patients with cerebral palsy versus the general population. Pediatr Pol. 2019;94(5):288-292; doi: 10.5114/polp.2019.89864.

48. Imanieh $\mathrm{MH}$, Golpayegan MR, Sedighi M, Ahmadi K, Aghaie A, Dehghani SM, et al. Comparison of three therapeutic interventions for chronic constipation in paediatric patients with cerebral palsy: a randomised clinical trial. Gastroenterology Rev. 2019;14(4):292-297; doi: 10.5114/pg.2019.84872. 